\title{
Integral reduction via algebraic curves
}

\author{
Yang Zhang* \\ ETH Zürich \\ E-mail: yang.zhangephys.ethz.ch

\section{Alessandro Georgoudis} \\ ETH Zürich \\ E-mail: georgouadstudent.ethz.ch
}

\begin{abstract}
We show that for a class of two-loop diagrams, the on-shell part of the integration-by-parts (IBP) relations correspond to exact meromorphic one-forms on algebraic curves. Since it is easy to find such exact meromorphic one-forms from algebraic geometry, this idea provides a new highly efficient algorithm for integral reduction. We demonstrate the power of this method via several complicated two-loop diagrams with internal massive legs. No explicit elliptic or hyperelliptic integral computation is needed for our method.
\end{abstract}

12th International Symposium on Radiative Corrections (Radcor 2015) and LoopFest XIV (Radiative Corrections for the LHC and Future Colliders)

15-19 June, 2015

UCLA Department of Physics \& Astronomy Los Angeles, USA

*Speaker. 


\section{Introduction}

The study of high precision scattering amplitudes in Quantum Chromodynamics and the Standard Model is important for the Run II of the Large Hadron Collider (LHC). However, the precise computation suffers from problems of the large number of loop Feynman diagrams and difficult loop integrations. This work aims at developing a new method of reducing loop integrals to the minimal set of integrals, i.e., master integrals (MIs).

Traditionally, integral reduction can be achieved by applying integration-by-parts (IBP) identities [1]. There are several implements of IBPs generating codes AIR [2], FIRE [3-5] and Reduze [6,7], based on Laporta algorithm [8], or LiteRed [9], based on a heuristic search of the symbolic IBP reduction. For multi-loop diagrams with high multiplicities or many mass scales, it may take a lot of computer time to finish the integral reduction. There are also several new approaches for integral reduction, based on the study of the Lie algebra structure of IBPs [10], Syzygy computation [11, 12], reductions over finite fields [13], and differential geometry [14]. Besides, the number of master integrals can be determined by the critical points [15].

We present a new method of integral reduction, for a class of multi-loop diagrams, based on unitarity [16-33] and the analysis of algebraic curves [34-36]. We show that for a $D$-dimensional $L$-loop diagram, if the unitarity cut solution $V$ is an irreducible algebraic curve, then the on-shell IBPs correspond to exact meromorphic 1-forms on $V$. For an algebraic curve, it is very easy to find the exact meromorphic 1-forms. Hence we get the on-shell IBP easily.

We consider some complicated diagrams to show the power of our method: (1) $D=4$ planar double box with internal massive legs. The unitarity cut for this diagram is an elliptic curve. (2) $D=4$ non-planar crossed box with internal massive legs. The unitarity cut for this diagram is a genus-3 hyperelliptic curve. For these examples, we get all the on-shell IBPs analytically. in the time order of minutes.

\section{Integral Reduction via the Analysis of Algebraic Curves}

Generically, for a quantum field theory, the $L$-loop amplitude can be written as $[16,17]$,

$$
A_{n}^{L-\text { loop }}=\sum_{k} c_{k} I_{k}+\text { rational terms }
$$

The set $\left\{I_{k}\right\}$ is called the master integral (MI) basis. Traditionally, the integral reduction is done by using IBP identities [1],

$$
\int \frac{d^{D} l_{1}}{(2 \pi)^{D}} \ldots \frac{d^{D} l_{L}}{(2 \pi)^{D}} \frac{\partial}{\partial l_{i}^{\mu}} \frac{v_{i}^{\mu}}{D_{1}^{\alpha_{1}} \ldots D_{k}^{\alpha_{k}}}=0
$$

if there is no boundary term.

We present a new way of integral reduction, based on maximal unitarity method and algebraic curves. Given a Feynman integral with $k$ propagators, maximal unitarity splits (2.1) as [18-33],

$$
\text { Int }=\sum_{i} c_{i} I_{i}+(\text { integrals with fewer-than-k propagators })+\text { rational terms }
$$

where the first sum is over the master integral with exact $k$ propagators. 
The condition the all internal legs are on-shell, is called the maximal unitarity cut,

$$
V: \quad D_{1}=\ldots=D_{k}=0,
$$

and the solution set for this equation system is an algebraic variety $V . V$ can be a set of discrete points, algebraic curves or surfaces. (See [37,38] for the detailed mathematical study.) Maximal unitarity replaces the original integral with contour integrals [26-33], schematically,

$$
\int \frac{d^{D} l_{1}}{(2 \pi)^{D}} \ldots \frac{d^{D} l_{L}}{(2 \pi)^{D}} \frac{N\left(l_{1}, \ldots l_{L}\right)}{D_{1}^{\alpha_{1}} \ldots D_{k}^{\alpha_{k}}} \rightarrow \oint \frac{d^{D} l_{1}}{(2 \pi)^{D}} \ldots \frac{d^{D} l_{L}}{(2 \pi)^{D}} \frac{N\left(l_{1}, \ldots l_{L}\right)}{D_{1}^{\alpha_{1}} \ldots D_{k}^{\alpha_{k}}}=\sum_{j} w_{j} \oint_{\mathscr{C}_{j}} \omega
$$

where $\omega$ is a differential form on $V$, and contours $c_{j}$ 's are around the poles of $\omega$ and also the nontrivial cycles of $V$ [30,32]. $w_{j}$ are weights of these contours. In particular, to extract the coefficients $c_{i}$ in (2.1), we can find a special set of weights $w_{j}^{\{i\}}[26-32]$ such that,

$$
c_{i}=\sum_{j} w_{j}^{\{i\}} \oint_{\mathscr{C}_{j}} \omega
$$

Our observation is that if a differential form $\omega$ on $V$ is integrated to zero, around all singular points on $V$, poles of $\omega$ and non-trivial cycles of $V$.

$$
\oint_{\mathscr{C}_{j}} \omega=0, \quad \forall j
$$

then from (2.6) and (2.3), the integral corresponding to $\omega$ can be reduced to integrals with fewer propagators.

We focus on the cases for which the number of propagators equals $D L-1$ and the maximal unitarity cut gives one irreducible variety. In such a case, the cut solution $V$ is a smooth algebraic curve with well defined complex structure. The condition (2.7) implies that $\omega$ is an exact meromorphic form on $V$, since the integral

$$
F(P)=\int_{O}^{P} \omega, \quad \forall P \in V
$$

is independent of the path and $d F=\omega$. Then from the study of meromorphic functions on $V$, we can list generators for $F$ and then derive all forms which satisfy (2.7). Explicitly, for this class of diagram, we find that the scalar integral on the cut becomes a holomorphic form on $V$.

$$
\left.\int \frac{d^{D} l_{1}}{(2 \pi)^{D}} \ldots \frac{d^{D} l_{L}}{(2 \pi)^{D}} \frac{1}{D_{1} \ldots D_{k}}\right|_{\text {cut }}=\oint \Omega
$$

where the 1-form $\Omega$ is globally holomorphic on $V$. On the cut, the components of $l_{i}$ 's become meromorphic functions. Let $F\left(l_{1}, \ldots l_{L}\right)$ be a polynomial in the components of loop momenta, then take the derivative of $F$,

$$
d F=f \Omega
$$

The resulting $f \Omega$ is an exact meromorphic 1 -form. From the analysis above, we get that,

$$
\int \frac{d^{D} l_{1}}{(2 \pi)^{D}} \cdots \frac{d^{D} l_{L}}{(2 \pi)^{D}} \frac{f}{D_{1} \ldots D_{k}}=0+\text { (integrals with fewer propagators) }
$$

so we obtain an integral reduction relation. 


\section{Elliptic Example: Double Box with Internal Masses}

The method explained in the previous section can be used for integral reduction for various topologies, for instance, the double-box (Fig. 1) with three different masses for the internal propagators.

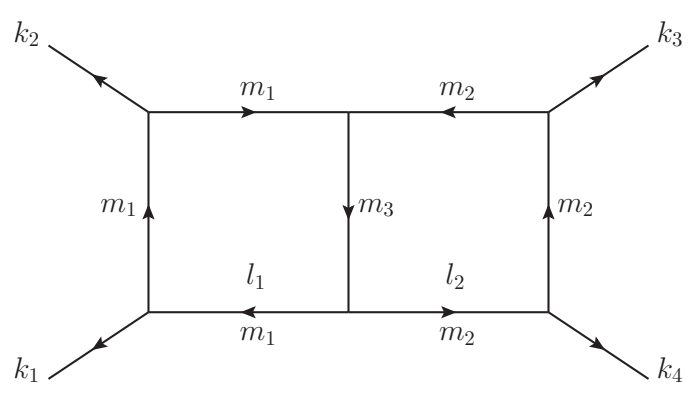

Figure 1: Planar double box diagram with 3 internal mass scales

\subsection{Maximal unitarity}

The denominators for double box diagrams are

$$
\begin{array}{lll}
D_{1}=l_{1}^{2}-m_{1}^{2}, & D_{2}=\left(l_{1}-k_{1}\right)^{2}-m_{1}^{2}, & D_{3}=\left(l_{1}-k_{1}-k_{2}\right)^{2}-m_{1}^{2}, \\
D_{4}=l_{2}^{2}-m_{2}^{2}, & D_{5}=\left(l_{2}-k_{4}\right)^{2}-m_{2}^{2}, & D_{6}=\left(l_{2}-k_{3}-k_{4}\right)^{2}-m_{2}^{2}, \\
D_{7}=\left(l_{1}+l_{2}\right)^{2}-m_{3}^{2} . &
\end{array}
$$

We parametrize the loop momenta as,

$$
\begin{aligned}
& l_{1}^{\mu}=\alpha_{1} k_{1}^{\mu}+\alpha_{2} k_{2}^{\mu}+\alpha_{3} \frac{s}{2} \frac{\left\langle 1\left|\gamma^{\mu}\right| 2\right]}{\langle 14\rangle[42]}+\alpha_{4} \frac{s}{2} \frac{\left\langle 2\left|\gamma^{\mu}\right| 1\right]}{\langle 24\rangle[41]}, \\
& l_{2}^{\mu}=\beta_{1} k_{3}^{\mu}+\beta_{2} k_{4}^{\mu}+\beta_{3} \frac{s}{2} \frac{\left\langle 3\left|\gamma^{\mu}\right| 4\right]}{\langle 31\rangle[14]}+\beta_{4} \frac{s}{2} \frac{\left\langle 4\left|\gamma^{\mu}\right| 3\right]}{\langle 41\rangle[13]} .
\end{aligned}
$$

The solutions for the maximal unitarity cut,

$$
D_{1}=D_{2}=\ldots=D_{7}=0
$$

defines an elliptic curve. To see this, we first solve for the variables $\alpha_{1}, \alpha_{2}, \alpha_{3}, \beta_{1}, \beta_{2}$ and $\beta_{3}$ in terms of $\alpha_{4}$ and $\beta_{4}$,

$$
\begin{array}{ll}
\alpha_{1}=1, & \alpha_{2}=0, \\
\beta_{1}=0, & \alpha_{3}=\frac{m_{1}^{2} t(s+t)}{\alpha_{4} s^{3}}, \\
\beta_{2}=1, & \beta_{3}=\frac{m_{2}^{2} t(s+t)}{\beta_{4} s^{3}},
\end{array}
$$

Then the remaining one equation relates $\alpha_{4}$ and $\beta_{4}$,

$$
K\left(\alpha_{4}, \beta_{4}\right)=A\left(\alpha_{4}\right) \beta_{4}^{2}+B\left(\alpha_{4}\right) \beta_{4}+C\left(\alpha_{4}\right)=0,
$$


Here $A\left(\alpha_{4}\right), B\left(\alpha_{4}\right)$ and $C\left(\alpha_{4}\right)$ are quadratic polynomials of $\alpha_{4}$, whose coefficients depend on kinematic variables. Formally, $\beta_{4}$ depends on $\alpha_{4}$ as,

$$
\beta_{4}=\frac{-B\left(\alpha_{4}\right) \pm \sqrt{\Delta\left(\alpha_{4}\right)}}{2 A\left(\alpha_{4}\right)}, \quad \Delta=B^{2}-4 A C
$$

where $\Delta$ is a quartic polynomial in $\alpha_{4}$ with four distinct roots. Hence the maximal unitarity cut defines an elliptic curve, i.e., algebraic curve with genus one,

On the cut, by a short calculation, the scalar double box integral on the cut, becomes

$$
\left.\int \frac{d^{4} l_{1}}{(2 \pi)^{4}} \frac{d^{4} l_{2}}{(2 \pi)^{4}} \frac{1}{D_{1} \ldots D_{7}}\right|_{\mathrm{cut}}=\frac{s^{2} t}{16} \oint \frac{d \alpha_{4}}{\sqrt{\Delta}},
$$

where the overall factor is not important for the following discussion. As [32], it is remarkable that $\frac{d \alpha_{4}}{\sqrt{\Delta}}$ is the only holomorphic one-form associated with the elliptic curve. On the cut, the loop-momentum components $\alpha_{i}, \beta_{i}$ become elliptic functions.

\subsection{Integral reduction}

We now focus on the double box integral with numerator $N$,

$$
I[N]=\int \frac{d^{4} l_{1}}{(2 \pi)^{4}} \frac{d^{4} l_{2}}{(2 \pi)^{4}} \frac{N}{D_{1} \ldots D_{7}},
$$

Integrand reduction method via Gröbner basis method $[39,40]$ determines that the integrand basis contains 32 terms. On the cut, the integral becomes a meromorphic one-form,

$$
\left.I[N]\right|_{\text {cut }} \propto \oint \frac{d \alpha_{4}}{\eta} N\left(\alpha_{3}, \alpha_{4}, \beta_{3}, \beta_{4}\right)
$$

where $N$ is a polynomial in $\alpha_{3}, \alpha_{4}, \beta_{3}$ and $\beta_{4}$, and therefore also an elliptic function. If two integrals on the cut, differ by the contour integrals of an exact meromorphic one-form $\omega$, then

$$
I\left[N_{1}\right]-\left.I\left[N_{2}\right]\right|_{\mathrm{cut}}=\oint \omega=0
$$

where the second equality holds for all contours, i.e., two fundamental cycles and small contours around the poles, because $\omega$ is exact. Then the integral reduction between $I\left[N_{1}\right]$ and $I\left[N_{2}\right]$ is achieved at the level of double box diagram,

$$
I\left[N_{1}\right]-I\left[N_{2}\right]=0+(\text { integrals with }<7 \text { propagators })
$$

Note the $\alpha_{4}$ and $\beta_{4}$ generate all elliptic functions on this elliptic curve. In practice, we find that to find such $\omega$ 's, it is sufficient to consider the exterior derivatives of polynomials in $\alpha_{3}, \alpha_{4}, \beta_{3}$ and $\beta_{4}$, So we need to find the one forms $\left\{d \alpha_{3}, d \alpha_{4}, d \beta_{3}, d \beta_{4}\right\}$ and then use the chain rule to generate integral reduction relations. We can start by calculating $d \alpha_{4}$ in terms of the holomorphic one-form,

$$
d \alpha_{4}=\eta \frac{d \alpha_{4}}{\eta}=\left(2 A\left(\alpha_{4}\right) \beta_{4}+B\left(\alpha_{4}\right)\right) \frac{d \alpha_{4}}{\eta}
$$


where we used the definition $\eta=\sqrt{\Delta}$ and (3.6) to rewrite $\eta$ in function of $\beta_{4}$. The purpose of this step is to get the a polynomial form of $f$.

We can now easily find $d \alpha_{3}$,

$$
d \alpha_{3}=d\left(\frac{\lambda_{1}}{\alpha_{4}}\right)=-\lambda_{1} \frac{1}{\alpha_{4}^{2}} d \alpha_{4}=-\frac{\alpha_{3}^{2}}{\lambda_{1}} d \alpha_{4}, \quad \lambda_{1} \equiv \frac{m_{1}^{2} t(s+t)}{s^{3}}
$$

To generate the remaining 1-forms, we again use the form of elliptic curve. Recall that,

$$
K\left(\alpha_{4}, \beta_{4}\right)=A\left(\alpha_{4}\right) \beta_{4}^{2}+B\left(\alpha_{4}\right) \beta_{4}+C\left(\alpha_{4}\right)=0 .
$$

The identity $d K=0$ reads,

$$
d \beta_{4}=-\left(A^{\prime}\left(\alpha_{4}\right) \beta_{4}^{2}+B^{\prime}\left(\alpha_{4}\right) \beta_{4}+C^{\prime}\left(\alpha_{4}\right)\right) \frac{d \alpha_{4}}{\eta} .
$$

Finally we can easily calculate $d \beta_{3}$,

$$
d \beta_{3}=d\left(\frac{\lambda_{2}}{\beta_{4}}\right)=-\lambda_{2} \frac{1}{\beta_{4}^{2}} d \beta_{4}=-\frac{\beta_{3}^{2}}{\lambda_{2}} d \beta_{4}, \quad \lambda_{2} \equiv \frac{m_{2}^{2} t(s+t)}{s^{3}}
$$

Then use the chain rule, we get all the on-shell IBPs. For example, we analytically obtain this relation,

$$
\begin{aligned}
I_{\mathrm{dbox}}\left[\alpha_{4}^{3}\right]= & \frac{1}{2 s^{4}\left(4 m_{2}^{2}-s\right)}\left(3 s^{3}\left(m_{1}^{2} s-m_{2}^{2} s-m_{3}^{2} s-4 m_{2}^{2} t+s t\right) I_{\mathrm{dbox}}\left[\alpha_{4}^{2}\right]\right. \\
+ & s\left(4 m_{1}^{2} s^{2} t-2 m_{2}^{2} s^{2} t-2 m_{3}^{2} s^{2} t+m_{1}^{4} s^{2}-2 m_{2}^{2} m_{1}^{2} s^{2}-2 m_{3}^{2} m_{1}^{2} s^{2}+m_{2}^{4} s^{2}+m_{3}^{4} s^{2}\right. \\
& \left.-2 m_{2}^{2} m_{3}^{2} s^{2}+2 m_{1}^{2} s t^{2}-4 m_{2}^{2} s t^{2}-8 m_{2}^{2} m_{1}^{2} s t-8 m_{2}^{2} m_{1}^{2} t^{2}+s^{2} t^{2}\right) I_{\mathrm{dbox}}\left[\alpha_{4}\right] \\
+ & \left.m_{1}^{2} t(s+t)\left(m_{1}^{2} s-m_{2}^{2} s-m_{3}^{2} s-4 m_{2}^{2} t+s t\right) I_{\mathrm{dbox}}[1]\right)+\ldots
\end{aligned}
$$

where ... stands for integrals with fewer than 7 propagators. Consider all polynomials whose exterior derivative satisfy the renormalizability conditions, we obtain 23 integral relations. Furthermore, consider Levi-Civita insertions which integrate to zero,

$$
\varepsilon\left(l_{2}, k_{1}, k_{2}, k_{3}\right) l_{2} \cdot k_{1}, \quad \varepsilon\left(l_{2}, k_{1}, k_{2}, k_{3}\right) l_{1} \cdot k_{4}, \quad \varepsilon\left(l_{1}, l_{2}, k_{1}, k_{2}\right), \quad \varepsilon\left(l_{1}, l_{2}, k_{1}, k_{3}\right) .
$$

we get 4 more integral relations. So the total number of MIs is \#MI $\mathrm{dbox}_{\mathrm{db}}=32-23-4=5$, and explicitly the MIs can be chosen as,

$$
\operatorname{MI}_{\mathrm{dbox}}=\left\{I_{\mathrm{dbox}}\left[\alpha_{4} \beta_{3}\right], I_{\mathrm{dbox}}\left[\alpha_{4}^{2}\right], I_{\mathrm{dbox}}\left[\alpha_{4}\right], I_{\mathrm{dbox}}\left[\beta_{3}\right], I_{\mathrm{dbox}}[1]\right\} .
$$

The whole computation takes about 120 seconds with our Mathematica code. The relations are numerically verified by FIRE [3,4].

\section{Hyperelliptic Example: Nonplanar Crossed Box with Internal Masses}

We now proceed in studying the integral reduction of the massive nonplanar double box (Fig. 2). Unlike the previous examples, this diagram's maximal unitarity cut provides a genus-3 hyperelliptic curve $[37,38]$. To illustrate our method, we consider the two-loop crossed box diagrams with massless external legs and three internel masses scales $\left\{m_{1}, m_{2}, m_{3}\right\}$. 


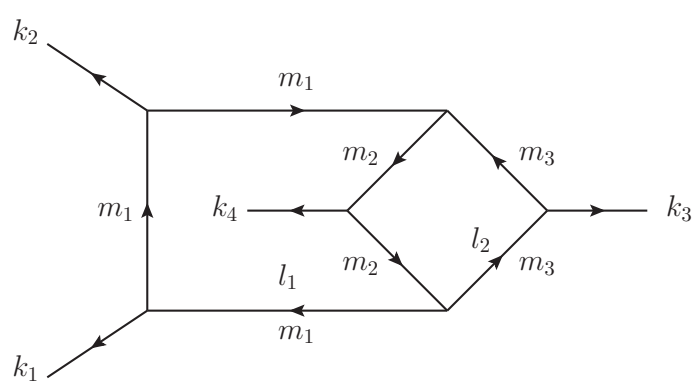

Figure 2: Nonplanar double box

\subsection{Maximal Unitarity and geometric properties}

The denominators for the Fig. 2 are,

$$
\begin{aligned}
& D_{1}=l_{1}^{2}-m_{1}^{2}, \quad D_{2}=\left(l_{1}-k_{1}\right)^{2}-m_{1}^{2}, \quad D_{3}=\left(l_{1}-k_{1}-k_{2}\right)^{2}-m_{1}^{2}, \\
& D_{4}=l_{2}^{2}-m_{3}^{2}, \quad D_{5}=\left(l_{2}-k_{3}\right)^{2}-m_{3}^{2}, \quad D_{6}=\left(l_{1}-l_{2}+k_{4}\right)^{2}-m_{2}^{2} \text {, } \\
& D_{7}=\left(l_{1}+l_{2}\right)^{2}-m_{2}^{2} \text {. }
\end{aligned}
$$

The on-shell constrains are $D_{1}=\ldots=D_{7}=0$. We use the same loop momenta parametrization (3.2). Again, we first solve for $\alpha_{1}, \alpha_{2}, \alpha_{3}, \beta_{1}, \beta_{2}$ and $\beta_{4}$ in terms of $\alpha_{4}$ and $\beta_{3}$,

$$
\begin{aligned}
& \alpha_{1}=1, \quad \alpha_{2}=0, \quad \alpha_{3}=\frac{m_{1}^{2} t(s+t)}{\alpha_{4} s^{3}}, \\
& \beta_{1}=-\left(\alpha_{4}+\alpha_{3}+\frac{t}{s}\right), \quad \beta_{2}=0, \quad \beta_{4}=\frac{\left(m_{3}^{2}\right) t(s+t)}{\beta_{3} s^{3}} .
\end{aligned}
$$

The rest two variables satisfy a polynomial equation,

$$
K\left(\alpha_{4}, \beta_{3}\right)=A\left(\alpha_{4}\right) \beta_{3}^{2}+B\left(\alpha_{4}\right) \beta_{3}+C\left(\alpha_{4}\right)=0,
$$

whose solution can be formally represented as,

$$
\beta_{3}=\frac{-B\left(\alpha_{4}\right) \pm \sqrt{\Delta\left(\alpha_{4}\right)}}{2 A\left(\alpha_{4}\right)}, \quad \Delta \equiv B^{2}-4 A C
$$

Unlike the previous examples, $\Delta\left(\alpha_{4}\right)$ here is a degree- 8 polynomial in $\alpha_{4}$ with 8 distinct roots. Hence the unitarity cut of this diagram provides a genus-3 hyperelliptic curve.

\subsection{Integral reduction}

First, the integrand reduction via Gröbner basis $[39,40]$ determines that, the integrand basis contains 38 terms in the numerator. Then, consider the maximal cut for the scalar integral of this diagram. The residue computation gives,

$$
\left.I_{\mathrm{xbox}}[1]\right|_{7-c u t}=\frac{s^{3}(s+t)}{16} \oint \frac{\alpha_{4} d \alpha_{4}}{\sqrt{\Delta\left(\alpha_{4}\right)}} .
$$


Note that unlike the elliptic case, on a genus-3 curve there are three holomorphic 1-forms.

$$
\frac{d \alpha_{4}}{\sqrt{\Delta\left(\alpha_{4}\right)}}, \quad \frac{\alpha_{4} d \alpha_{4}}{\sqrt{\Delta\left(\alpha_{4}\right)}}, \quad \frac{\alpha_{4}^{2} d \alpha_{4}}{\sqrt{\Delta\left(\alpha_{4}\right)}}
$$

the scalar integral cut corresponds to the second one.

This hyperelliptic curve have 6 fundamental cycles and 8 poles as shown in the previous subsection. By global residue theorem, only 7 poles' residues are independent. Therefore we may perform maximal unitarity by computing integrals over $6+7=13$ contours. Therefore the number of master integers must be 13 .

Following what we did for elliptic cases, we calculate the differential forms $\left\{d \alpha_{3}, d \alpha_{4}, d \beta_{3}, d \beta_{4}\right\}$.

$$
d \alpha_{4}=\frac{\eta}{\eta} d \alpha_{4}=\left(2 A\left(\alpha_{4}\right) \beta_{4}+B\left(\alpha_{4}\right)\right) \frac{d \alpha_{4}}{\eta}=\left(2 A\left(\alpha_{4}\right) \beta_{4}-B\left(\alpha_{3}\right)\right) \frac{\alpha_{3}}{\lambda_{1}} \frac{\alpha_{4} d \alpha_{4}}{\eta},
$$

where we have used the usual definition $\eta \equiv \sqrt{\Delta}$. In the second equality, we used the on-shell identity, $\alpha_{3} \alpha_{4}=\lambda_{1} \equiv \frac{m_{1}^{2} t(s+t)}{s^{3}}$ to recover the form of the scalar integral cut (4.5). The step is not needed for elliptic cases. Then,

$$
d \alpha_{3}=d\left(\frac{\lambda_{1}}{\alpha_{4}}\right)=-\lambda_{1} \frac{1}{\alpha_{4}^{2}} d \alpha_{4}=-\frac{\alpha_{3}^{2}}{\lambda_{1}} d \alpha_{4}
$$

where again we have used (4.2) to simplify our expression. The exterior derivatives for $\beta_{i}$ are more complicated,

$$
d \beta_{3}=-\left(A^{\prime}\left(\alpha_{4}\right) \beta_{3}^{2}+B^{\prime}\left(\alpha_{4}\right) \beta_{3}+C^{\prime}\left(\alpha_{4}\right)\right) \frac{\alpha_{3}}{\lambda_{1}} \frac{\alpha_{4} d \alpha_{4}}{\eta},
$$

and,

$$
d \beta_{4}=d\left(\frac{\lambda_{2}}{\beta_{3}}\right)=-\lambda_{2} \frac{1}{\beta_{3}^{2}} d \beta_{3}=-\frac{\beta_{4}^{2}}{\lambda_{2}} d \beta_{3}, \quad \lambda_{2}=\frac{m_{3}^{2} t(s+t)}{s^{3}}
$$

Given a polynomial function of $\left\{\alpha_{i}, \beta_{i}\right\}$, we can use the chain rule to generate the on-shell IBPs.

Again, we also consider Levi-Civita insertions. In total, we generate 25 on-shell IBPs and 6 Levi-Civita insertions identities. Hence there are 38-25-6=7 MIs for the non-planar crossed box diagram with three internal mass scales. Define that $X=\left(l_{1}+p_{4}\right)^{2} / 2$ and $Y=\left(l_{2}+p_{1}\right)^{2} / 2$, and the MIs can be chosen as:

$$
\left\{I_{\text {xbox }}\left[X^{3}\right], I_{\text {xbox }}\left[Y^{2}\right], I_{\text {xbox }}[X Y], I_{\text {xbox }}\left[X^{2}\right], I_{\text {xbox }}[X], I_{\text {xbox }}[Y], I_{\text {xbox }}[1]\right\} .
$$

For this non-planar diagram, the analytic integral reduction relations are significantly more complicated. The integral reduction at the level of crossed box takes about 22 minutes with our Mathematica code, and the relations obtained by our method have been numerically verified by FIRE $[3,4]$.

\section{Outlooks}

Beyond the cases discussed, it would be interesting to study the $\varepsilon$-dependent part of the integral reduction, based on our method. One apparent difficulty is that the spacetime dimension is not a constant, so the cut solution's dimension or geometric structure is not fixed. This problem can be solved by using the dimension-dependent measure and dimension-independent propagators in the integrand. The research on two-loop maximal unitarity in dimensional regularization scheme [41], also based on algebraic geometry tools, help us to understand IBPs with dimensional regularization from a geometric viewpoint. 


\section{References}

[1] K.G. Chetyrkin and F.V. Tkachov. Integration by Parts: The Algorithm to Calculate beta Functions in 4 Loops. Nucl.Phys., B192:159-204, 1981.

[2] Charalampos Anastasiou and Achilleas Lazopoulos. Automatic integral reduction for higher order perturbative calculations. JHEP, 0407:046, 2004.

[3] A.V. Smirnov and V.A. Smirnov. FIRE4, LiteRed and accompanying tools to solve integration by parts relations. Comput.Phys.Commun., 184:2820-2827, 2013.

[4] A.V. Smirnov. Algorithm FIRE - Feynman Integral REduction. JHEP, 0810:107, 2008.

[5] A.V. Smirnov. An Algorithm to construct Grobner bases for solving integration by parts relations. JHEP, 0604:026, 2006.

[6] A. von Manteuffel and C. Studerus. Reduze 2 - Distributed Feynman Integral Reduction. 2012.

[7] C. Studerus. Reduze-Feynman Integral Reduction in C++. Comput.Phys.Commun., 181:1293-1300, 2010.

[8] S. Laporta. High precision calculation of multiloop Feynman integrals by difference equations. Int.J.Mod.Phys., A15:5087-5159, 2000.

[9] R. N. Lee. Presenting LiteRed: a tool for the Loop InTEgrals REDuction. 2012.

[10] R.N. Lee. Group structure of the integration-by-part identities and its application to the reduction of multiloop integrals. JHEP, 0807:031, 2008.

[11] Janusz Gluza, Krzysztof Kajda, and David A. Kosower. Towards a Basis for Planar Two-Loop Integrals. Phys.Rev., D83:045012, 2011.

[12] Robert M. Schabinger. A New Algorithm For The Generation Of Unitarity-Compatible Integration By Parts Relations. JHEP, 1201:077, 2012.

[13] Andreas von Manteuffel and Robert M. Schabinger. A novel approach to integration by parts reduction. Phys. Lett., B744:101-104, 2015.

[14] Yang Zhang. Integration-by-parts identities from the viewpoint of differential geometry. 2014.

[15] Roman N. Lee and Andrei A. Pomeransky. Critical points and number of master integrals. JHEP, $11: 165,2013$.

[16] Zvi Bern, Lance J. Dixon, David C. Dunbar, and David A. Kosower. One loop n point gauge theory amplitudes, unitarity and collinear limits. Nucl.Phys., B425:217-260, 1994.

[17] Zvi Bern, Lance J. Dixon, David C. Dunbar, and David A. Kosower. Fusing gauge theory tree amplitudes into loop amplitudes. Nucl.Phys., B435:59-101, 1995.

[18] Ruth Britto, Freddy Cachazo, and Bo Feng. Generalized unitarity and one-loop amplitudes in N=4 super-Yang-Mills. Nucl.Phys., B725:275-305, 2005.

[19] Ruth Britto, Freddy Cachazo, and Bo Feng. New recursion relations for tree amplitudes of gluons. Nucl.Phys., B715:499-522, 2005.

[20] Ruth Britto, Freddy Cachazo, and Bo Feng. Computing one-loop amplitudes from the holomorphic anomaly of unitarity cuts. Phys. Rev., D71:025012, 2005.

[21] Ruth Britto, Freddy Cachazo, Bo Feng, and Edward Witten. Direct proof of tree-level recursion relation in Yang-Mills theory. Phys.Rev.Lett., 94:181602, 2005. 
[22] Ruth Britto, Evgeny Buchbinder, Freddy Cachazo, and Bo Feng. One-loop amplitudes of gluons in SQCD. Phys.Rev., D72:065012, 2005.

[23] Charalampos Anastasiou, Ruth Britto, Bo Feng, Zoltan Kunszt, and Pierpaolo Mastrolia. D-dimensional unitarity cut method. Phys.Lett., B645:213-216, 2007.

[24] Charalampos Anastasiou, Ruth Britto, Bo Feng, Zoltan Kunszt, and Pierpaolo Mastrolia. Unitarity cuts and Reduction to master integrals in d dimensions for one-loop amplitudes. JHEP, 0703:111, 2007.

[25] Freddy Cachazo. Holomorphic anomaly of unitarity cuts and one-loop gauge theory amplitudes. 2004.

[26] David A. Kosower and Kasper J. Larsen. Maximal Unitarity at Two Loops. 2011.

[27] Henrik Johansson, David A. Kosower, and Kasper J. Larsen. Two-Loop Maximal Unitarity with External Masses. Phys.Rev., D87:025030, 2013.

[28] Mads Sogaard. Global Residues and Two-Loop Hepta-Cuts. 2013.

[29] Henrik Johansson, David A. Kosower, and Kasper J. Larsen. Maximal Unitarity for the Four-Mass Double Box. 2013.

[30] Simon Caron-Huot and Kasper J. Larsen. Uniqueness of two-loop master contours. JHEP, 1210:026, 2012.

[31] Mads Søgaard and Yang Zhang. Multivariate Residues and Maximal Unitarity. JHEP, 12:008, 2013.

[32] Mads Søgaard and Yang Zhang. Elliptic Functions and Maximal Unitarity. Phys.Rev., D91(8):081701, 2015.

[33] Henrik Johansson, David A. Kosower, Kasper J. Larsen, and Mads Søgaard. Cross-Order Integral Relations from Maximal Cuts. Phys. Rev., D92(2):025015, 2015.

[34] P. Griffiths and J. Harris. Principles of Algebraic Geometry. Wiley Classics Library. Wiley, 2011.

[35] R. Miranda. Algebraic Curves and Riemann Surfaces. Dimacs Series in Discrete Mathematics and Theoretical Comput. American Mathematical Society, 1995.

[36] H.M. Farkas and I. Kra. Riemann Surfaces: With 27 Figures. Graduate Texts in Mathematics. Springer New York, 1992.

[37] Rijun Huang and Yang Zhang. On Genera of Curves from High-loop Generalized Unitarity Cuts. JHEP, 1304:080, 2013.

[38] Jonathan D. Hauenstein, Rijun Huang, Dhagash Mehta, and Yang Zhang. Global Structure of Curves from Generalized Unitarity Cut of Three-loop Diagrams. JHEP, 02:136, 2015.

[39] Yang Zhang. Integrand-Level Reduction of Loop Amplitudes by Computational Algebraic Geometry Methods. JHEP, 1209:042, 2012.

[40] Pierpaolo Mastrolia, Edoardo Mirabella, Giovanni Ossola, and Tiziano Peraro. Scattering Amplitudes from Multivariate Polynomial Division. Phys.Lett., B718:173-177, 2012.

[41] Kasper J. Larsen and Yang Zhang. Integration-by-parts reductions from unitarity cuts and algebraic geometry. 2015. 